\title{
Research on dark noise features of CMOS image sensor Lingxiao $\mathrm{Li}^{1,}$ a , Jinho $\mathrm{Kim}^{2}$, Guangmang $\mathrm{Cui}^{1}$, Zhihai $\mathrm{Xu}^{1,} \mathrm{~b}^{*}$, Huajun Feng ${ }^{1}$, Qi $\mathrm{Li}^{1}$, Yueting Chen ${ }^{1}$ \\ ${ }^{1}$ Department of State Key Laboratory of Modern Optical Instrumentation, Zhejiang University, Hangzhou, 310027, China \\ ${ }^{2}$ Department of Samsung Electronics, Kiheung, Korea \\ aemail: lilingxiao1@126.com, bemail: xuzh@zju.edu.cn
}

Keywords: CMOS image sensor; Dark noise; Time distribution; Spatial distribution

\begin{abstract}
In this paper, the noises of images taken by Cannon40D and CMOS industrial camera are analyzed both in time and space domain without outside illumination. The dark random noise associated with time is very low, which means the system noise is almost uncorrelated with time. However, system noise in the center of the CMOS image sensor is lower than those in the marginal area, which suggests the noise presents a certain spatial distribution. Finally, taking the temperature effect into consideration, we find the relationship between the maximum noise and temperature.
\end{abstract}

\section{Introduction}

Compared with CCD sensor, CMOS has many advantages such as mature craft, low price, low power consumption and high integration [1], which would become the tendency of the future image sensor. CIS could be applied to the areas of digital camera, video device, security monitoring and medical equipment.

Dark noise is a significant aspect in CMOS application. Dark noise is mainly caused by dark current non-uniformity and device defects. Many researchers have conducted relevant work. C.M.Hu [3] studied the flicker noise in the CMOS device; Min H.S. [5] researched the model of the temporal pixel to pixel noise of CMOS Image Sensors; Wang [4] analyzed the Random Telegraph Signal (RTS) in CIS pixels.

The rest of the papers are organized as follows. Firstly we make a brief introduction about the theory and structure of CIS. And then the detailed methods are proposed to deal with features of dark noise in different conditions. Finally, we provide the analysis of time-domain and spatial domain characteristics as well as the temperature experimental results of CIS dark noise.

\section{CIS working principle}

CIS belongs to active pixel sensor, the process of photon that diverts into electron and transfer electric circuits are both achieved in the image unit [2]. A complete CIS unit circuit is shown in Fig.1.

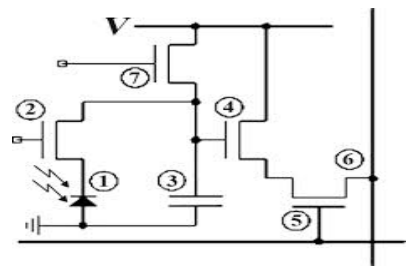

Fig.1. CMOS structure

In Fig.1, (1) represents the photosensitive diode (PD). It changes the received photon into electron in the exposure process. (7)(4)(5) are MOS transistors, (7) is used as reset switch. When reset impulse comes, (7) will open and transfer the voltage of PD into the reset level; (4)is used as source follower (SF), it aims to buffer the voltage and amplify the current. (5) is used as output switch. When addressing impulse comes, it gates the voltage of SF into the buffer and amplifies the 
output. The process of CIS is as follows: Photon signal is diverted into the electric current through the photosensitive unit, and then changed into the digital image signal with the process of latter amplification and $\mathrm{AD}$ transfer. In this process, a variety of noise will be inevitably brought in, which leads to the image quality decrease.

From the CIS working process above, we can see with the drive of addressing impulse, the electrical signal which is diverted from photon of PD will directly output and go on the next process such as amplification and AD transfer. Therefore, the image noise doesn't mainly occur in the process of charge transfer, but comes from latter amplification circuit. Because every CMOS image unit has its own electrical processing circuit, and the electrical signal will fluctuate with time, therefore different image units have different noise distributions which are associated with the time and space.

\section{Time-domain analysis of CIS noise}

In this section we conduct an experiment to analysis CIS noise in time domain. Two types of CMOS camera are selected in our experiment, including Cannon 40D and a CMOS industrial camera. The pixel resolutions of Cannon 40D and CMOS industrial camera are $1936 \times 1288$ and $2048 \times 1536$, respectively. In order to analysis the dark noise caused by CIS instead of the imaging system, imaging lens is removed and cameras are placed in the total dark environment. In this way several raw data images are captured to calculate the dark noise distribution. In the experiment 100 dark images are captured for each camera. To illustrate the dark noise performance relatived with time, the noise of each pixel for the whole frame is obtained by calculating the standard deviation of 100 dark noise images [3], as the equation shows:

$\sigma=\sqrt{\frac{1}{n} \sum_{i=1}^{n}\left(x_{i}-\bar{x}\right)^{2}}$

Where $\sigma$ is the standard deviation, in our case $n=100 . x_{i}$ is the value of each pixel, $\bar{x}$ is the average value, the value of $\mathrm{Y}$ label is the logarithm of the number of each $\sigma$ which represents the dark random noise. The overall distributions of the two cameras are shown in Fig.2.

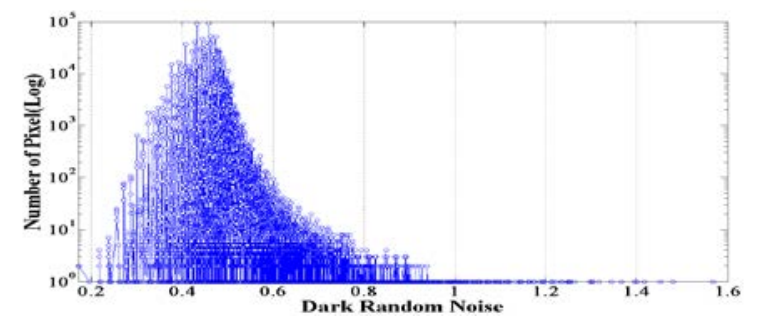

(a)Noise distribution of Cannon40D

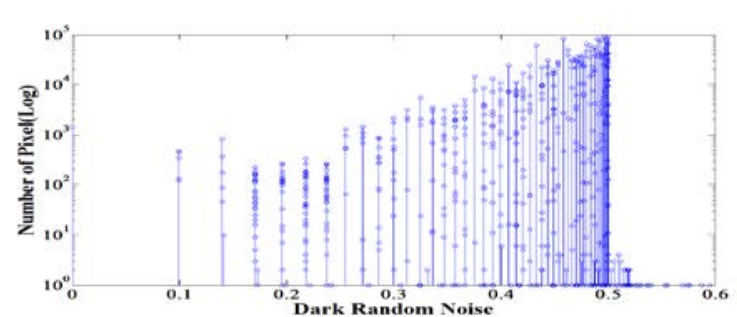

(b) Noise distribution of CMOS industrial camera

Fig.2. Noise distributions of CMOS camera

In Fig.2, it can be seen that the dark random noise levels associated with time for both CMOS cameras are relatively low. Most of the standard deviation statistical values are around 0.5. For Cannon 40D, the noise distribution is more like a Gaussian-like distribution figure. While for the CMOS industrial camera, more noise pixels distributes in the low noise level range. For both the types of CMOS cameras, the dark random noise in time domain has little real effect on the final image output.

\section{Spatial-domain analysis of CIS noise}

Though CIS unit and circuit are all made with MOS technique in the same chip, each pixel has a corresponding signal output and amplifying circuit [4]. Therefore, different areas have different noise distributions. We adopted two ways to analyze the spatial-domain distribution of the CIS noise. Firstly we select five different location patches of the Cannon40D camera. These patches are the four corners and the center of the sensor, respectively, as shown in Fig.3. They are chosen to represent the noise distribution of whole frame. 
All of the patches are with the pixel size of $100 \times 100$. For each patch, we calculate every pixel value and draw the color distribution figure, which can directly reflect the CIS noise uniformity in each patch. The color distribution figures are shown in Fig. 4.
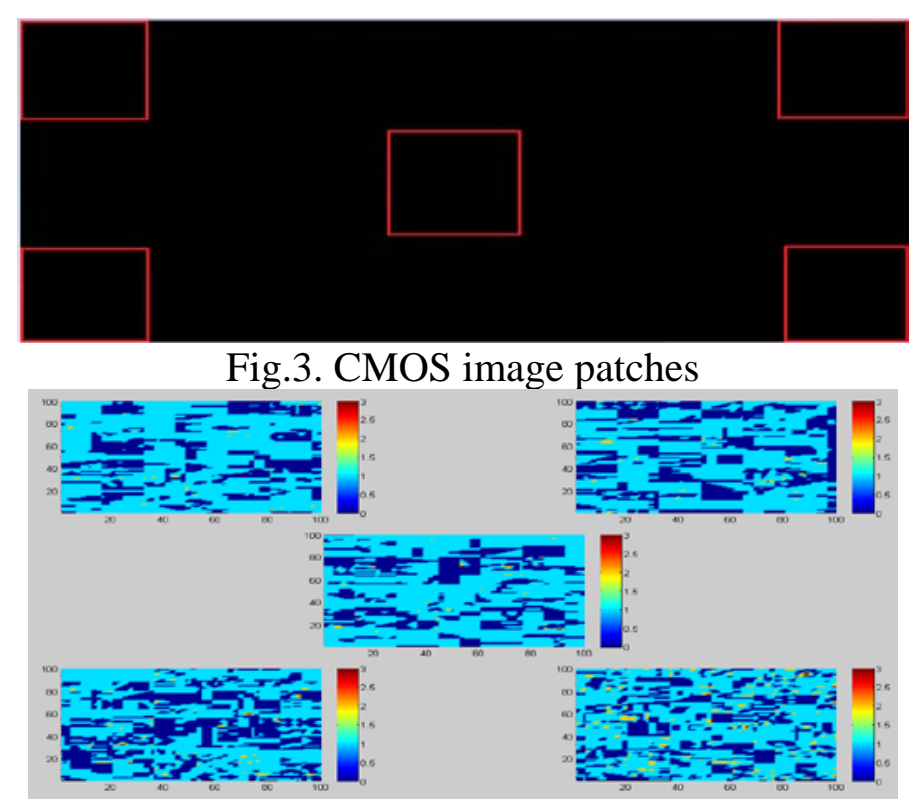

Fig.4. CMOS color distribution curve

In Fig.4, different colors represent the different pixel value. The cold color reflects the low pixel values, while the warm color reflects the relative large pixel noise values, as the illustration in the colorbar. From the results we can see that the patches at the edge of image have a higher noise level than the patch located in the center. The distribution of the spatial-domain noise in a single CMOS image is non-uniform. It may be caused by the CMOS manufacture procedure. For CMOS industrial camera, as shown in Fig. 2(b), the noise distribution is more uniform and smooth. In this case, we consider utilizing the local average pixel value to reflect its spatial distribution. Similar with Fig.3, we divide the image into several patches, including the corner patches and center patch and calculate their average pixel values. For 100 images, we can obtain 100 values of each patch, and draw their corresponding average distribution curves, as shown in Fig.5.

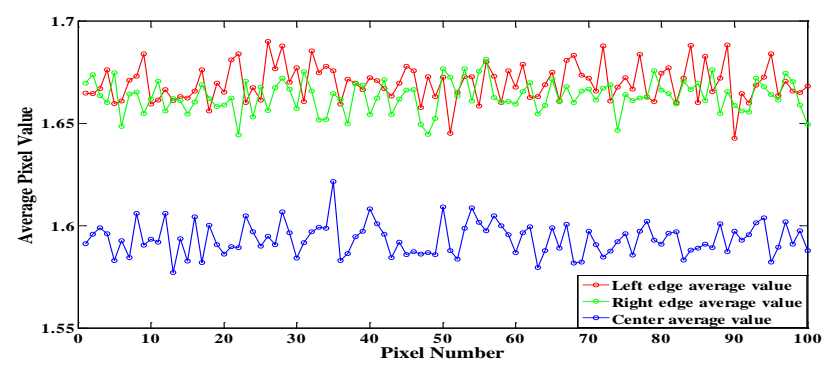

Fig.5. CMOS average value distribution

In Fig. 5 we can see average value of the center is visibly lower than the edge value. It is the same as Fig.4. In short, we can draw the conclusion that the CIS noise presents a certain spatial distribution, the noise level in the middle pixel of the image is lower than those at the edge.

\section{Temperature experiments}

For CIS, the dark noise is sensitive to the temperature no matter the inherent or readout noise [5]. Therefore we also conduct the experiments to analyze the relationship between the noise and temperature. We put the CMOS industrial camera in a thermostat, and gradually change the environment temperature from $0^{\circ} \mathrm{C}$ to $40^{\circ} \mathrm{C}$. In different temperature conditions we make the camera exposed the same way as before and analyze the captured dark image. We draw the 3D color distribution curve to reflect the noise changes and compare the differences between different 
temperatures. The results are shown in Figure 6 (a), (b) and (c).

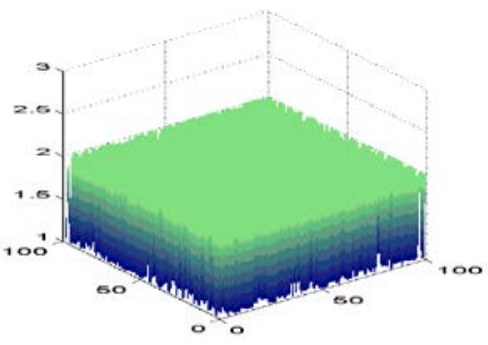

(a) Noise distribution at $0^{\circ} \mathrm{C}$

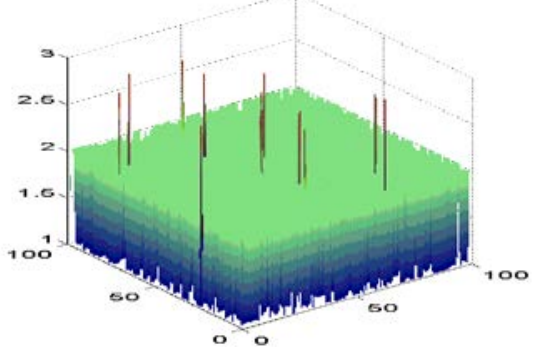

(b) Noise distribution at $20^{\circ} \mathrm{C}$

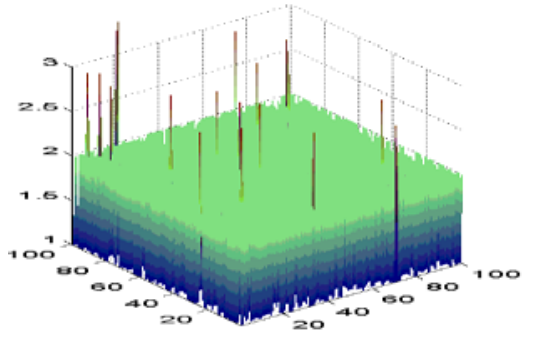

(c) Noise distribution at $40^{\circ} \mathrm{C}$

Fig.6. Color distribution curve in different temperature

In Fig. 6 we can see the maximum noise value will increase with the temperature increases, this result is consistent with what we have expected. Generally, the power spectral density (PSD) of the thermal noise is proportional to the temperature [6], which means the thermal noise will increase with the temperature rise. Therefore, the temperature is indeed an important factor which has an impact on the CIS noise.

\section{Conclusion}

This paper analyzed the noise features of CMOS image sensor based on dark exposure and data processing. During the experiment, first we find that the dark current associated with time has little effect on the CIS noise distribution, which means the CIS noise is almost uncorrelated with time. However, the CIS noise presents a certain spatial distribution, the noise in the middle of the image is lower than those at the edge. Finally, according to the temperature experiments, it's obvious that with the rise of temperature, the maximum noise will increase.

\section{Acknowledgement}

This work is supported by National Natural Science Foundation of China (Grant no. 61275021) and Samsung's program of Evaluation and Improvement for the Performance of CMOS Imaging Sensor(Grant no. I20140064).

\section{References}

[1] Luo Bin, Yang Fu-xing, Yan Lei. Key technologies and research development of CMOS image sensors. Geoscience and Remote Sensing (IITA-GRS), 2010 Second IITA International Conference[C], 2010 322(325), 28-31.

[2] Yuan Yu-lei, Zou Liang, Ai Jin-peng. Analysis of CMOS image sensor noise without outside illumination. Engineering of Surveying and Mapping[J], 2011 20(4), 31-34.

[3] Hung K K, Ko P K, Chenming H, et al. A unified model for the flicker noise in metal oxide semiconductor field-effect transistors[J]. Electron Devices, IEEE Transactions on. 1990, 37(3): 654-665.

[4] Wang X, Rao P R, Mierop A, et al. Random telegraph signal in CMOS image sensor pixels[C]. San Francisco, CA, United states: Institute of Electrical and Electronics Engineers Inc., 2006.

[5] Jun-Myung W, Hong-Hyun P, Min H S, et al. Statistical analysis of random telegraph noise in CMOS image sensors[C]. Hakone: 2008.

[6] Woo J, Park H, Hong S, et al. Statistical Noise Analysis of CMOS Image Sensors in Dark Condition[J]. IEEE Transactions on Electron Devices. 2009, 56(11): 2481-2488. 